\title{
EFEITOS ADVERSOS IDENTIFICADOS EM LOCAL DE INFUSÃO INTRAVENOSA PERIFÉRICA POR DROGAS QUIMIOTERÁPICAS •
}

\author{
ADVERSE EFFECTS IDENTIFIED AT THE LOCATION OF PERIPHERAL \\ INTRAVENOUS INFUSION BY CHEMOTHERAPY DRUGS
}

\section{EFECTOS ADVERSOS POR DROGAS QUIMIOTERÁPICAS IDENTIFICADOS EN EL SITIO DE INFUSIÓN INTRAVENOSA PERIFÉRICA}

\author{
Paula Elaine Diniz dos Reis **, Cíntia Capucho Rodrigues ${ }^{* * *}$, Christiane Inocêncio \\ Vasques****, Emília Campos de Carvalho*****
}

\begin{abstract}
RESUMO
A rede endovenosa é uma das principais vias para administração de quimioterápicos e requer um cuidado especial na prevenção de extravasamento. Este estudo tem como objetivo verificar a incidência de alterações locais na rede venosa de indivíduos em tratamento oncológico por quimioterapia. Trata-se de um estudo observacional longitudinal, com 15 sujeitos, maiores de 18 anos, sendo 11 do sexo feminino. Foram observadas 20 punções venosas, realizadas predominantemente em membros superiores $(\mathrm{n}=19)$. Antes da quimioterapia o local de acesso apresentava-se íntegro $(n=18)$, rede venosa com trajeto tortuoso $(n=11)$ e de pequeno calibre $(\mathrm{n}=11)$. A maioria das punções foi realizada por enfermeiros, com dispositivo intravenoso de agulha rígida $(n=19)$ calibre $23(n=12)$. Durante a punção houve verbalização de dor $(n=2)$, queimação $(n=3)$ e ardência $(n=1)$ ao longo do trajeto venoso; as manifestações locais observadas foram eritema $(n=1)$, edema $(n=5)$ e extravasamento $(n=3)$. Imediatamente após a remoção do dispositivo de infusão ocorreram eritema $(n=3)$, hematoma $(n=1)$. Tardiamente houve hematoma $(n=5)$, eritema $(n=2)$, fleboesclerose $(n=2)$ e verbalização de dor no local $(n=1)$. A identificação dos efeitos adversos relacionados à infusão endovenosa periférica de quimioterapicos torna possível o desenvolvimento de estratégias de assistência de enfermagem capazes de reduzir e prevenir tais danos ao paciente.
\end{abstract}

Palavras chave: Cateterismo periférico, infusões intravenosas, efeitos adversos, enfermagem, cuidados de enfermagem.

- Trabalho desenvolvido no Grupo de Pesquisa Enfermagem e Comunicação da Escola de Enfermagem de Ribeirão Preto - EERP-USP. Subsidiado pelo CNPq.

${ }^{* *}$ Enfermeira, Doutoranda da EERP-USP, bolsista do CAPES.

${ }^{* * *}$ Enfermeira, Mestranda da EERP-USP

${ }^{* * * *}$ Enfermeira, Doutoranda da EERP-USP, bolsista do CAPES.

${ }^{* * * * *}$ Enfermeira, Professora Titular do Departamento de Enfermagem Geral e Especializada da EERP-USP. Correspondência: Emília Campos de Carvalho. E-mail: ecdcava@usp.br . Av Bandeirante 3900 CEP 14040-902 Ribeirão Preto-SP 


\begin{abstract}
The intravenous system is one of the main chemotherapy administration routes and requires special care to prevent extravasations. This study aims to verify the incidence of local changes in the venous network of cancer patients under chemotherapy. This observational and longitudinal study involves 15 patients over 18 years old, 11 of whom were women. Twenty venous punctures were observed, mostly in the superior limbs $(n=19)$. Before chemotherapy, the access location was found intact $(n=18)$, tortuous venous system $(n=11)$ of small caliber $(\mathrm{n}=11)$. Most punctures were performed by nurses, using a peripheral intravenous device with a rigid needle $(n=19)$, caliber $23(n=12)$. During the puncture, there was verbalization of pain $(n=2)$, burning $(n=3)$ and stinging $(n=1)$ along the venous pathway; erythema $(n=1)$, edema $(n=5)$ and extravasation $(n=3)$ were observed as local manifestations. Immediately after the removal of the infusion device, erythema $(n=3)$ and hematoma $(n=1)$ were observed. Later on, hematoma $(n=5)$, erythema $(n=2)$, phlebosclerosis $(n=2)$ and verbalization of pain in the infusion site $(n=1)$ occurred. Identifying the adverse effects related to the peripheral intravenous infusion of chemotherapy makes it possible to develop nursing care strategies that can reduce and prevent this damage in patients.
\end{abstract}

Keywords: Catheterization, peripheral; infusions, Intravenous; adverse effects; nursing; nursing care.

\title{
RESUMEN
}

La vía endovenosa es una de las principales vías para la administración de quimioterápicos y requiere un cuidado especial en la prevención de la extravasación. Este estudio tiene por objetivo verificar la incidencia de alteraciones locales en la red venosa de individuos en tratamiento oncológico con quimioterapia. Este estudio tiene característica observacional y longitudinal, con 15 personas mayores de 18 años, siendo 11 del sexo femenino. Fueron realizadas 20 punciones venosas, la mayor parte en miembros superiores $(n=19)$. Antes de la quimioterapia, el sitio de acceso se presentaba íntegro $(n=18)$, red venosa con trayecto tortuoso $(n=11)$ y de pequeño calibre $(n=11)$. La mayor parte de las punciones fue realizada por enfermeros, utilizando un dispositivo intravenoso periférico de aguja rígida $(n=19)$, calibre $23(n=12)$. Durante la punción se registró verbalización de dolor $(\mathrm{n}=2)$, quema $(\mathrm{n}=3)$ y ardor $(\mathrm{n}=1)$ a lo largo del trayecto venoso; las manifestaciones locales observadas fueron eritema $(n=1)$, edema $(n=5)$ y extravasación $(n=3)$. Inmediatamente después de retirar el dispositivo de infusión se manifestó eritema $(n=3)$ y hematoma $(n=1)$. Tardíamente se registró hematoma $(n=5)$, eritema $(n=3)$, fleboesclerosis $(n=2)$ y verbalización de dolor $(n=1)$. La identificación de los efectos adversos relacionados a la infusión endovenosa periférica de quimioterápicos permite desarrollar estrategias de atención de enfermería capaces de disminuir y prevenir tales daños al paciente.

Palabras clave: Cateterismo periférico, infusiones intravenosas; efectos adversos, enfermería, atención de enfermería

Fecha Recepción: 28 febrero 2008 Fecha Aceptación: 02 octubre 2008

\section{INTRODUÇÃO}

O câncer, de um modo genérico, pode ser definido como uma doença na qual há multiplicação e disseminação pelo organismo de formas celulares anormais. É a segunda causa de morte por doenças no Brasil, subseqüentemente às doenças cardiovasculares (Ministerio da Saude de Brasil, 2005).

Existem, atualmente, quatro possíveis for- mas de abordagem no tratamento de uma neoplasia maligna: excisão cirúrgica, irradiação, quimioterapia e terapia biológica. A escolha dentre essas opções depende do tipo de neoplasia, do estágio em que se encontra seu desenvolvimento e das especificidades celulares do tumor.

Dentre as modalidades terapêuticas, destaca-se a quimioterapia que consiste no emprego de substâncias químicas, isoladas 
ou em combinação, que atuam diretamente nas células, interferindo no processo de crescimento e divisão celular, bem como em micrometástases, impossíveis de serem detectadas por exames clínico, radiológico e cintilográfico.

Os quimioterápicos podem ser classificados, de acordo com sua ação a nível celular, em drogas ciclo celular específico e drogas ciclo celular não específico. Bonassa y Santana (2005) referem que as drogas ciclo celular específico são mais ativas no combate às células que se encontram em uma determinada fase do ciclo, em geral fase síntese $(\mathrm{S})$ ou mitose (M). Assim sendo, são efetivas no tratamento dos tumores de divisão rápida $\mathrm{e}$ ativa. Já as drogas ciclo celular não específico são letais às células em qualquer fase do ciclo, pois atuam sobre a fração proliferativa e não proliferativa do tumor. De acordo com sua estrutura química e função a nível celular, podem ser divididos em sete grandes grupos: agentes alquilantes, antimetabólitos, antibióticos antitumorais, agentes hormonais, alcalóides da vinca, nitrosuréias e miscelânea.

A quimioterapia, embora tenha se mostrado efetiva no tratamento das doenças neoplásicas, apresenta vários efeitos colaterais por atuar em todas as células do organismo que se encontram em fase de divisão celular; contudo ainda é considerada viável devido a rápida e total recuperação dos tecidos sadios frente às células tumorais (Ministerio da Saude de Brasil, 2008).

Existem diversas vias para administração de quimioterapia, a saber: oral, intramuscular, subcutânea, endovenosa, intrarterial, intratecal, intraperitoneal, intrapleural, intravesical, intra-retal e aplicação tópica. A via endovenosa é considerada a via de administração mais segura no que se refere à absorção e manutenção do nível sérico da droga. $\mathrm{O}$ acesso a ela pode ser feito por meio de punção de veia periférica ou através de cateteres, tanto de curta quanto de longa permanência (Bonassa \& Santana, 2005).

Entretanto, cuidados especiais devem ser dispensados quando da utilização dessa via de administração, principalmente devido à toxicidade local, vascular e dermatológica, causada por grande parte dos quimioterápicos. Dentre os sinais e sintomas que caracterizam tal toxicidade figuram o desconforto passageiro no local de punção, vasoespasmo, dor, eritema, flebite e necrose tecidual severa, comprometendo nervos e tendões, ocasionada pelo extravasamento de drogas vesicantes no tecido vizinho à veia puncionada (Phillips, 2005), podendo chegar até a sensação de morte eminente (Wilkes, 2008).

Há dois grupos de agentes antineoplásicos que causam alterações locais: o das drogas irritantes, que quando infiltradas nos tecidos, fora do trajeto venoso, causam desconforto local ao longo da veia associado à hiperemia local; e o grupo das drogas vesicantes, cuja infiltração nos tecidos, fora do trajeto venoso, leva a fixação da droga ao DNA da célula produzindo lesão celular imediata, provocando irritação severa, podendo formar vesículas e subseqüente necrose tecidual (Oestreicher, 2007).

$\mathrm{Na}$ prática de enfermagem observam-se situações em que estão presentes fatores de risco ou etiológicos de danos à rede venosa ou das estruturas próximas a ela, isto é, lesando diretamente a estrutura do vaso ou de tecidos vizinhos, decorrente quer da presença do dispositivo de infusão ou de líquidos infundidos ou drenados Tais alterações são denominadas de risco de trauma vascular ou trauma vascular, respectivamente (Arreguy-Sena \& Carvalho, 2007; ArreguySena, 2002). Dentre as causas, destacam-se o contato de líquidos (vesicantes ou não vesicantes) com o espaço extra-vascular ou epiderme, o uso do mesmo sítio para múltiplas punções ou em pequenos intervalos de tempo, garroteamento por tempo excessivo, correlação indevida entre calibre do vaso e do dispositivo empregado, excesso de circuito sem fixação efetiva, transfixação de vasos ou deslocamento da agulha no interior do vaso, tempo prolongado de uso do mesmo 
sitio, excesso de adesivos nas áreas adjacentes aos dispositivos, falta de oclusão eficaz, umidade ou sujidade nesses locais (Maki \& Ringer, 1991; Hadaway, 2002; Phillips, 2005; Bonassa\& Santana, 2005; Arreguy-Sena \& Carvalho, 2007). Freqüentes trombocitopenias e fragilidade vascular devido ao déficit nutricional em decorrência da doença também foram apontadas por Bonassa y Santana (2005) como responsáveis pela ocorrência de tais danos.

Dada a relevância de se identificar fatores de risco ou etiológicos dessas lesões a fim de que possam ser estabelecidas metas de redução desses indicadores, realizou-se o presente estudo, cujo objetivo foi verificar a ocorrência de alterações locais (tromboflebite, dor, eritema, vasoespasmo, vesículas e necrose tecidual) na rede venosa periférica de indivíduos adultos submetidos à quimioterapia endovenosa.

\section{MATERIAL E MÉTODOS}

O presente estudo, de caráter descritivo, longitudinal, foi realizado em uma instituição hospitalar filantrópica, com atendimento multidisciplinar a indivíduos portadores de neoplasias malignas em regime de Hospital-Dia (HD); contando com 10 leitos e 03 poltronas para atender os usuários (ambos os sexos e diferentes faixas etárias) agendados previamente. A equipe de enfermagem responsável pelo atendimento era formada por seis profissionais: três enfermeiras e três auxiliares de enfermagem. O projeto foi aprovado pelo Comitê de Ética em Pesquisa da Escola de Enfermagem de Ribeirão PretoUSP.

\section{Sujeitos:}

Uma amostra de conveniência foi composta por indivíduos atendidos na referida instituição para realização de quimioterapia endovenosa periférica. Foram incluídos aqueles que atendiam aos seguintes critérios: -maio- res de 18 anos; diagnóstico de neoplasia maligna; -em tratamento quimioterápico por via endovenosa periférica, único ou em concomitância com outra modalidade de terapêutica não endovenosa (como por exemplo, a radioterapia) desde que esta não estivesse sendo realizada nos membros superiores (sítio de possível punção venosa periférica) no período de coleta dos dados; -atendidos no HD pelo menos duas vezes no período em que os dados foram coletados; -não estivessem recebendo nenhuma outra medicação endovenosa por via periférica no sítio de punção da quimioterapia, entre os períodos de atendimentos no HD em que estava sendo realizada a coleta de dados, quer seja através de atendimentos em instituições hospitalares com regime de internação convencional, ou em serviços de emergência, domiciliar e Unidades Básicas de Saúde; -concordassem em participar do estudo, externando seu aceite por meio da assinatura do "Termo de Consentimento Livre e Esclarecido Pós-Informado" de acordo com a Resolução 196/96 do Ministério da Saúde - Brasil.

\section{Procedimentos:}

A coleta de dados foi realizada, por uma das pesquisadoras, nas dependências do $\mathrm{HD}$, após a assinatura do termo de consentimento livre esclarecido com as devidas elucidações sobre sigilo, anonimato e livre participação no estudo.

A pesquisa consistiu da observação do sítio de punção do indivíduo em três momentos, a saber: antes da inserção do cateter para a punção venosa periférica a fim de caracterizar a integridade da rede venosa e da pele do mesmo; no momento da punção venosa para registrar o tipo de procedimento (técnica da punção) e o material utilizado na execução desta e, posteriormente (uma hora após a infusão e no retorno, até 30 dias), para identificar possíveis alterações na rede venosa, subseqüentes à administração da quimioterapia endovenosa.

$\mathrm{O}$ instrumento de coleta de dados utiliza- 
do era composto de questões abertas e fechadas. Estas permitiram caracterizar a amostra quanto à idade, sexo e patologia; e ainda, registrar os dados obtidos pelos métodos de mensuração e de observação, por meio das técnicas semiológicas (inspeção e palpação), sobre o local de inserção do cateter endovenoso periférico e áreas adjacentes, em plano superficial e profundo. Tal observação foi realizada em quatro momentos distintos, a saber: antes da inserção do dispositivo, durante a infusão da medicação, no período pós imediato (até 1 hora após a remoção do dispositivo) e tardio (no próximo retorno). As alterações estudadas foram: trobomflebite, dor, eritema, vasoespasmo, vesículas e necrose tecidual.

\section{Resultados}

\section{Caracterização da amostra}

A amostra foi constituída por 15 sujeitos, sendo 11 mulheres e quatro homens, cuja idade média apresentada foi de 55,3 anos.

Dentre as neoplasias que acometeram os indivíduos do sexo masculino, 02 sujeitos apresentavam câncer de intestino (adenocarcinoma de cólon), 01 câncer de laringe e 01 câncer de estômago. No sexo feminino houve predomínio de neoplasias do ovário e hematológicas contando, com 03 sujeitos cada uma delas, seguidas pelas neoplasias de mama e intestino com 02 sujeitos cada; identificou-se ainda a de pulmão com 01 sujeito.

\section{Caracterização do sítio venoso}

A avaliação da pele realizada imediatamente antes do procedimento de punção venosa evidenciou que a maioria dos sujeitos $(\mathrm{n}=13)$ apresentava pele íntegra no sítio de punção; os demais apresentavam escoriações e descamações. As características relacionadas à coloração da pele e turgor também foram avaliadas e estão descritas na Tabela 1.

Tabela 1: Distribuição das características do sítio de punção de pacientes submetidos à infusão endovenosa periférica de drogas quimioterápicas segundo a coloração da pele e turgor.

\begin{tabular}{|c|c|c|c|}
\hline \multirow{2}{*}{\multicolumn{2}{|c|}{ Variáveis }} & \multicolumn{2}{|c|}{ Freqüência } \\
\hline & & $\mathrm{N}$ & $\%$ \\
\hline Coloração & $\begin{array}{l}\text { corada } \\
\text { descorada } \\
\text { hiperemiada } \\
\text { ictérica }\end{array}$ & $\begin{array}{l}08 \\
09 \\
03 \\
01\end{array}$ & $\begin{array}{c}38 \\
43 \\
14,3 \\
4,7\end{array}$ \\
\hline Turgor & $\begin{array}{l}\text { normal } \\
\text { diminuído }\end{array}$ & $\begin{array}{l}04 \\
16\end{array}$ & $\begin{array}{l}20 \\
80\end{array}$ \\
\hline
\end{tabular}

*de acordo com classificação adotada por Porto (2001)

Em relação à localização da punção, apenas um sujeito foi puncionado no membro inferior. As punções em membro superior ocorreram nas veias do dorso da mão $(n=12)$ e da face anterior do antebraço $(n=7)$. Cabe destacar que quatro sujeitos foram puncionados mais de uma vez, totalizando 20 punções. As avaliações do trajeto, calibre e visualização da rede venosa desses sujeitos estão descritas na Tabela 2, porém duas punções não puderam ser avaliadas quanto a esses critérios devido a impossibilidade de visualização e palpação da mesma no local de punção. Houve garroteamento do membro puncionado em todos os procedimentos, sendo que o tempo não foi superior a dois minutos em $85 \%$ deles. 
Tabela 2: Distribuição das características da rede venosa de pacientes submetidos à infusão endovenosa periférica de drogas quimioterápicas segundo trajeto, calibre e visualização.

\begin{tabular}{|c|c|c|c|c|}
\hline \multirow{2}{*}{\multicolumn{2}{|c|}{ Variáveis }} & \multicolumn{3}{|c|}{ Freqüência } \\
\hline & & $\mathrm{N}$ & $\%$ & Total \\
\hline Trajeto & $\begin{array}{l}\text { Tortuoso } \\
\text { Retilíneo }\end{array}$ & $\begin{array}{l}11 \\
09\end{array}$ & $\begin{array}{l}55 \\
45\end{array}$ & 100 \\
\hline Calibre & $\begin{array}{l}\text { Pequeno } \\
\text { Médiano } \\
\text { Grande } \\
\text { Sem avaliação }\end{array}$ & $\begin{array}{l}11 \\
06 \\
01 \\
02\end{array}$ & $\begin{array}{l}55 \\
30 \\
05 \\
10\end{array}$ & 100 \\
\hline Visualizaçãa* & $\begin{array}{l}\text { V/P } \\
\text { V/NP } \\
\text { NV/P } \\
\text { NV/NP }\end{array}$ & $\begin{array}{l}08 \\
08 \\
02 \\
02\end{array}$ & $\begin{array}{l}40 \\
40 \\
10 \\
10\end{array}$ & 100 \\
\hline
\end{tabular}

*V/P - visível e palpável; V/NP - visível e não palpável; NV/P - não visível e palpável; $\mathrm{NV/NP}$ - não visível e não palpável

Em relação ao dispositivo intravenoso utilizado, foi empregado o dispositivo de agulha rígida em 19 das vinte punções. Quanto ao calibre, o número 21 foi utilizado em 06 punções, o número 23 em 11 e o número 25 em 01. A única punção realizada com dispositivo intravenoso flexível utilizou o calibre 24. Destaca-se que todos os dispositivos foram fixados por adesivo tipo micropore, geralmente com dois ou três fragmentos do material localizados sobre o dispositivo, perpendicularmente à inserção do mesmo.

O álcool a $70 \%$ foi utilizado para realização da antissepsia da pele em todas as punções. A fim de manter a permeabilidade do acesso venoso e retirada de resíduos de quimioterápicos foi utilizado soro fisiológico a $0,9 \%(n=14)$ e soro glicofisiológico $(n=6)$, antes e após a infusão de cada um dos agentes quimioterápicos.

\section{Efeitos Adversos Identificados}

Durante a administração dos quimioterápicos os principais efeitos adversos verbalizados pelos sujeitos foram dor $(\mathrm{n}=2)$ e queimação $(n=3)$ ao longo do trajeto venoso e ardência no local de punção venosa $(n=1)$. Frente aos eventos descritos, além de obser- var atentamente o local de punção venosa, a conduta tomada pela equipe de enfermagem foi a realização de manobras para teste de refluxo sangüíneo e diminuição da velocidade do gotejamento.

Quando observado o local de acesso venoso durante a infusão do quimioterápico, as manifestações identificadas foram eritema local $(n=1)$, edema $(n=5)$ e extravasamento $(n=3)$. Nestas situações, a equipe de enfermagem optou pela retirada do dispositivo venoso. Nos casos de extravasamento, inicialmente realizou-se a aspiração do conteúdo extravasado e posterior aplicação de antídotos, previamente estabelecidos em protocolo pela instituição.

Foram observados, após o término da infusão e retirada do dispositivo venoso, as seguintes reações locais: eritema $(n=3)$ e hematoma acompanhado de edema $(n=1)$. Tardiamente, foram identificados hematoma $(n=5)$, eritema $(n=2)$, fleboesclerose $(n=2)$ e dor no membro $(n=1)$.

\section{Discussão}

\section{Caracterização dos sujeitos}

Segundo Ministerio da Saude de Brasil (2008), o câncer é um problema de saúde 
pública neste país devido ao envelhecimento da população, dentre outros fatores, pois favorece maior exposição a fatores ambientais e mudanças comportamentais que facilitam o desenvolvimento de neoplasias.

De acordo com as estimativas do Ministério da Saúde de Brasil (2007), os tipos de câncer mais incidentes na população masculina são os de pele não melanoma, próstata, pulmão e estômago. Nas mulheres, destacam-se as neoplasias de pele não melanoma, mama, colo de útero, cólon e reto, respectivamente. Embora o tamanho da amostra limite análises de cunho epidemiológico, é possível afirmar que as neoplasias identificadas nos participantes deste estudo reiteram os dados supracitados.

\section{Caracterização do sítio:}

As observações realizadas no local de punção, previamente ao procedimento, evidenciaram condições hígidas dos vasos sanguíneos. Apenas 10\% dos sujeitos apresentavam escoriações e descamações cutâneas, possivelmente relacionadas ao efeito colateral dos agentes quimioterápicos que estes haviam recebido..

Apesar de 65\% apresentarem alterações na coloração da pele, esta é uma condição freqüente na população, devido à toxicidade hematológica causada pelos quimioterápicos.

Outra alteração observada foi em relação ao turgor da pele, sendo $20 \%$ dos sujeitos classificados como turgor diminuído. Segundo Smeltzer y Bare (2005), a diminuição da turgidez da pele pode estar relacionada com o déficit de volume líquido corporal devido à redução da ingestão hídrica ou eliminação por perda ativa associada às náuseas e vômitos, efeitos colaterais comuns na população em estudo.

No presente estudo, observou-se que a maior parte das punções foram realizadas em membros superiores. As recomendações da literatura são imperativas ao afirmar que se deve puncionar, preferencialmente, as veias dos membros superiores para a administração de quimioterápicos. Aquelas localizadas nos membros inferiores raramente devem ser usadas devido ao alto risco de ocorrência de tromboembolismo, salvo em situações especiais. Mesmo quando se punciona veias em membros superiores, algumas ações devem ser evitadas, a saber: puncionar veias abaixo de uma infiltração endovenosa prévia ou área flebítica; veias excessivamente puncionadas, esclerosadas ou trombosadas; membros que tenham derivação ou fístula arteriovenosa, acometido por edema, metástases, infecção, dilaceração da pele; membro homolateral ao esvaziamento linfático axilar devido ao comprometimento do retorno venoso, ou ainda, com distúrbios motores e/ou sensoriais como plegia, paresia e parestesia (Bonassa \& Santana, 2005; Smeltzer \& Bare, 2005; Hadaway, 2004).

Os dados apontam que apenas $5 \%$ dos vasos puncionados eram de grande calibre. Além disso, menos da metade das veias apresentavam-se visíveis e palpáveis no momento da punção. Bonassa \& Santana (2005) recomenda a punção de veias de médio a grande calibre, pouco tortuosas e móveis, evitando aquelas que se apresentam rígidas, endurecidas, doloridas ou com alterações de cor.

Os pacientes submetidos a tratamento oncológico, freqüentemente, apresentam rede venosa precária, dificultando a punção. Os dados apresentados demonstram que o dispositivo endovenoso periférico com agulha rígida, calibre 23 , foi o mais empregado para punção. Hadaway (2004) e Oestreicher (2007) sugerem que o uso de cateteres periféricos de menos calibrosos provocam menor trauma ao vaso, reduzindo o risco de extravasamento; além de assegurar maior fluxo sanguíneo em torno do cateter, o que promove a hemodiluição das drogas infundidas.

Em contraposição, Nascimento y Souza (1996) aponta que o calibre do dispositivo não interfere significativamente na ocorrência de infiltrações no local de punção. Tais 
autores, no entanto, concordam que o uso de agulhas rígidas oferece maior risco de extravasamento quando comparado aos dispositivos flexíveis. Entretanto, também há que se considerar o tempo de infusão na escolha do dispositivo. Phillips (2005) e Honório e Nascimento (2007) indicam o uso de agulha rígida de aço apenas quando o tempo de infusão é curto (poucas horas) e o volume infundido é pequeno.

$\mathrm{O}$ índice de sucesso na primeira punção, outro aspecto observado, foi de $55 \%$, no presente estudo. A literatura orienta que devam ser feitas no máximo duas tentativas para punção venosa a fim de evitar trauma desnecessário ao paciente e a limitação do acesso vascular (Phillips, 2005). Somente em duas situações foram necessárias mais de duas punções em um mesmo paciente.

Quanto ao dispositivo, a literatura recomenda que o mesmo deva ser fixado de modo que não interfira com a visualização e avaliação do local, além de que a adequada fixação reduz o risco de complicações relacionadas à terapia intravenosa (Phillips, 2005; Hadaway, 2004). O material utilizado, adesivo tipo micropore, e a forma de fixação adotada mostraram-se adequados no presente estudo.

A bomba de infusão contínua (BIC), também denominada Controlador de Fluxo, opera estritamente sob o fluxo de gravidade e reduz o potencial de infusão rápida de grandes quantidades de solução, além de auxiliar a equipe de enfermagem na detecção de infiltrações sinalizando com som de alarme quando ocorre resistência do fluxo (Phillips, 2005). Este dispositivo foi empregado em $80 \%$ das punções, embora tenha se observado o uso concomitante de outros tipos de equipo para infusão na maioria dos casos.

Para avaliar a permeabilidade da veia é recomendado que se infunda de 10 a $20 \mathrm{ml}$ de solução salina a 0,9\% antes de administrar um agente citotóxico e que, posteriormente à infusão do antineoplásico, injete-se soro fisiológico a $0,9 \%$ para retirada de resíduos da droga da periferia (Phillips, 2005). Os fluidos isotônicos geralmente não causam tanto desconforto quanto os hipertônicos ou com pH ácido ou alcalino (Millan, 1988). No presente estudo, em nenhum momento, durante a infusão do fluido não concomitantemente ao quimioterápico, foi verbalizado dor ou desconforto pelos pacientes.

\section{Efeitos Adversos no sítio de infusão venosa}

Os dados evidenciam que $40 \%$ dos sujeitos apresentavam queixas referidas (de dor, queimação ou ardência) ou danos observados (eritema local, edema ou extravasamento). Tais ocorrências se deram em (45\%) das punções realizadas. Neste aspecto a literatura aponta que de 20 a $80 \%$ dos pacientes que recebem terapia intravenosa periférica desenvolvem flebite. (Panadero, Iohom, Taj, Mackay \& Shorten, 2002).

Até que se prove o contrário, uma queixa de dor ou queimação deve ser sempre considerada como sintoma de extravasamento até que se prove o contrário (Phillips, 2005). No presente estudo, a retirada do dispositivo venoso, pela equipe de enfermagem, foi realizada em sete situações. Em cinco delas houve apenas a remoção do dispositivo: uma associada à presença de dor, queimação e edema; outra em caso de edema; outra ainda relacionada a ardência e edema; outra em queimação e eritema e, por fim, em uma situação de edema e extravasamento. Nas outras duas situações, a remoção da punção foi precedida por aspiração e aplicação de antídotos.

Entretanto, sintomas de dor ou queimação, embora presentes, podem representar não extravasamento, desde que confirmado por meio de manobras de refluxo e de avaliação do local. Em tais situações o dispositivo tem sido mantido; isso se observou em dois casos no presente estudo, nos quais os dispositivos foram mantidos até o final do procedimento, embora os pacientes relatassem queimação $(n=1)$ e dor $(n=1)$ não sendo referida ou observada nenhuma outra evidência. A conduta de manter o cateter é 
sustentada pela literatura; as escala de flebite de Jackson (1998) e da INS (2000) consideram haver flebite grau 1 na presença da constatação de eritema ou vermelhidão próximo ao sítio de inserção e, só então, é recomendado a retirada do dispositivo.

A patologia e o tipo de ciclo prescrito, parece que não interferiram nos achados; embora considerando que todas as pacientes que apresentaram danos vasculares eram mulheres, elas apresentaram diagnósticos médicos distintos (Cancer de ovário, de pulmão, de mama, mieloma múltiplo e doença de Hodking) e, portanto protocolos diferentes; também eram de idades distintas, variando de 33 a 67 anos.

Em comum apresentavam punções em veias tortuosas, a maioria $(\mathrm{n}=7)$ de pequeno calibre, todas visíveis e a maioria $(\mathrm{n}=7)$ não palpável. Cinco veias estavam localizadas no dorso da mão e três na face anterior do antebraço. A solução que precedia e se seguia à quimioterapia também parece não ter sido fator determinante para as ocorrências.

Considerando-se os danos observados, estratégias de enfermagem podem ser aplicadas para reduzi-los ou elimina-los. Nesse sentido, uso de cateter de longa permanência pode ser útil para o paciente, reduzindo dor e desconforto, bem como para a equipe de enfermagem, pois esse dispositivo auxilia na instalação da quimioterapia, facilitando-a, e ampliando a segurança do paciente uma vez que disponibiliza maior calibre para acesso intravenoso.

Cabe lembrar, finalmente, que o tamanho da amostra não permite fazer outras abordagens estatísticas, com vistas a indicar fatores preditivos de risco de lesão ou de lesão vascular. Nesse sentido, novos estudos deverão ser desenvolvidos.

\section{REFERÊNCIAS}

Arreguy-Sena, C. \& Carvalho, E.C.C. (2007) Trauma vascular: proposição dos elementos do diagnóstico e validação do conteúdo por peritos. Pensar Enfermagem, 11(1): 12-23.

Arreguy-Sena, C. (2002). A trajetória de construção e validação dos diagnósticos de enfermagem: Trauma Vascular e Risco para Trauma Vascular. [Tese] Ribeirão Preto(SP). Escola de Enfermagem de Ribeirão Preto- USP. 280pp.

Bonassa, E.M.A.\& Santana, T (2005) Enfermagem em terapêutica oncológica. ( $3^{\mathrm{a}}$ ed.) São Paulo: Atheneu. 538pp.

Hadaway, L.C. (2002). IV infiltration: not just a peripheral problem. Nursing, 32(8),36-43.

Hadaway, L.C. (2004). Preventing and managing peripheral extravasation. Nursing, 34(5),66-7.

Honório, M.O. \& Nascimento, K.C. (2007). Acessos venosos periféricos. Núcleo de educação em urgência. Florianópolis.disponível em http//neu.saude.sc.gob.br/arquivos/ acessos_venosos_periféricos.pdf. Recuperado el 05/01/2008

Intravenous Nursing Society. (2000). Infusion nursing standards of practice. $J$ Intraven Nurs., 23 (6S):44-50.

Jackson, A. (1998). Infection control: a battle in vein infusion phlebitis. Nursing times, 94(4):68-71.

Lewis, C.B.H.; Hecker, J.F. (1985). Infusion thrombophlebitis. Br. J. Anaesth, 57: 220-33.

Maki, D.G.\& Ringer, M. (1991).Risk factors for infusion-related phlebitis with small peripheral venous catheters. Ann. Intern. Med., 114, 845-854.

Millan, D.A. (1988). Managing complications of IV therapy. Nursing, 18(3), 34-43.

Ministério da Saúde de Brasil (2007). Estimativa da incidência de câncer no Brasil. Rio de Janeiro. Disponível em: http://www.inca.gov.br/ estimativa/2008. Recuperado el 23/01/2008.

Ministério da Saúde de Brasil (2005) Informações de saúde. Sistema de Informação sobre Mortalidade. Disponível em: http://w3.datasus. gov.br/datasus. Recuperado el 10/01/2008.

Ministério da Saúde de Brasil (2002). Ações de enfermagem para o controle do câncer: uma proposta de integração ensino - serviço. (2.ed.). Rio de Janeiro: INCA. 376 pp.

Ministério da Saúde de Brasil.. (2008). Conselho Nacional de Saúde. Resolução 196 de 10 de outubro de 1996. Diretrizes e normas regulamentadoras da pesquisa envolvendo seres humanos. Brasília, 1996. 
Nascimento, E.M.F.\& Souza, M.F. (1996). Infiltração em terapia intravenosa através de veia periférica. Act. Paul. Enf., 9(1),53-60.

Panadero, A.; Iohom, G.; Taj, J.; Mackay, N.; Shorten, G. (2002). A dedicated intravenous cannula for postoperative use Effect on incidence and severity of phlebitis. Anaesthesia. 57, 921-25.

Phillips, L.D. (2005). Manual of IV Therapeutics. ( $4^{\mathrm{a}}$ ed). Davis: Philadelphia. 812 pp.

Porto, C.C. (2001). Exame Clínico. Bases para a prática médica. $4^{\text {a }}$ Ed. Rio de Janeiro: Guanabara Koogan,. 439 p.
Smeltzer, S.C.\& Bare, B.G. (2005). Brunner \& Suddarth tratado de enfermagem médicocirúrgica. $10^{\text {a }}$ ed. Rio de Janeiro: Guanabara Koogan. 1679 pp.

Oestreicher, P. (2007). Can you recognize the risk factors for vesicant extravasation? ONS Connect.22(1):22-3.

Wilkes, G.(2008). Managing drug infusion reactions: focus on cetuximab monoclonal antibody therapy. Clinical Journal of Oncology Nursing, 12 (3): 530-2. 